\title{
The matrix element for radiative Bhabha scattering in the forward direction
}

\author{
Ronald Kleiss \\ NIKHEF-H \\ Amsterdam, The Netherlands
}

\begin{abstract}
We present an approximation to the matrix element for the process $e^{+} e^{-} \rightarrow e^{+} e^{-} \gamma$, appropriate to the situation where one or both of the fermions are scattered over very small angles. The leading terms in the situation where all scattering angles are small contains not only terms quadratic in the electron mass, but also quartic and even sextic terms must be included. Special attention is devoted to the numerical stability of the resultant expression. Its relation to several existing formulae is discussed.
\end{abstract}

For any $e^{+} e^{-}$collider, the process of Bhabha scattering is very important. Whereas large-angle scattering provides its share of information on QED or theories of electroweak interactions, small-angle scattering serves as a tool for accurate luminosity determination; in each of these cases, radiative corrections (and hence bremsstrahlung) cannot be neglected. But the radiative process by itself, namely

$$
e^{+}\left(p_{1}\right) e^{-}\left(q_{1}\right) \rightarrow e^{+}\left(p_{2}\right) e^{-}\left(q_{2}\right) \gamma(k),
$$

is important in its own right, especially when one or either of the electrons is scattered over a small, or even negligible, angle. For instance, such events form an important background to low-energy photon-photon processes, as recently pointed out, for the DA $\Phi$ NE collider, in [1]; moreover, interestingly, the process (1) is, for machines such as LEP, the most important restriction on the beam lifetimes (see, for instance, [2]). Since the Bhabha cross 
section peaks at small fermion scattering angles, and the photon is preferentially emitted more or less collinearly with a charged particle, such processes are dominated by events where all scattering angles are small, and many different momentum transfers become small simultaneously. It is therefore important to have reliable expressions for the matrix element (we shall always understood it to be squared and summed/averaged over all particle spins) in these extreme kinematical configurations. An allowed simplification of the problem, if we restrict ourselves to small fermion scattering angles, is to neglect the $s$-channel diagrams, and the interference between radiation from the positron and that from the electron line. Hence, in what follows we shall assume that we only have the two Feynman diagrams in which the photon is emitted by the incoming and outgoing positron (radiation from the electron is then trivially obtained by $e^{+} \leftrightarrow e^{-}$). We introduce the following notation:

$$
\begin{aligned}
P^{\mu} & =p_{1}^{\mu}+q_{1}^{\mu}=p_{2}^{\mu}+q_{2}^{\mu}+k^{\mu} \\
q^{\mu} & =q_{1}^{\mu}-q_{2}^{\mu} \\
s & =P \cdot P \equiv 4 E^{2} \\
t & =q \cdot q \\
\Delta_{1,2} & =p_{1,2} \cdot k \\
s_{1} & =\left(p_{2}+q_{2}\right)^{2}=(P-k)^{2} .
\end{aligned}
$$

The five invariants $s, s_{1}, \Delta_{1,2}$, and $t$, specify the kinematics completely up to a trivial azimuthal rotation around the beam axis. Using the algebraic program FORM [3], we then find for the matrix element, $|\mathcal{M}|^{2}$ :

$$
\begin{aligned}
\frac{1}{e^{6}}|\mathcal{M}|^{2}=\frac{1}{\Delta_{1} \Delta_{2}(-t)} & {\left[\left(s-6 m^{2}\right)^{2}+\left(s+4 m^{2}-2 \Delta_{2}+t\right)^{2}\right.} \\
& +\left(s_{1}-6 m^{2}\right)^{2}+\left(s_{1}+4 m^{2}+2 \Delta_{1}+t\right)^{2} \\
& \left.-12 m^{2}\left(t+10 m^{2}\right)\right] \\
+\frac{8 m^{2}}{\Delta_{1} \Delta_{2} t^{2}} & {\left[\left(s-2 m^{2}-2 \Delta_{2}+t\right)\left(s_{1}-2 m^{2}-2 \Delta_{1}+t\right)\right.} \\
& \left.-\left(\Delta_{1}-\Delta_{2}\right)^{2}\right] \\
- & \frac{2 m^{2}}{\Delta_{1}^{2} t^{2}}\left[\left(s_{1}-2 m^{2}\right)^{2}+\left(s_{1}-2 m^{2}+t\right)^{2}+4 m^{2} t\right]
\end{aligned}
$$




$$
-\frac{2 m^{2}}{\Delta_{2}^{2} t^{2}}\left[\left(s-2 m^{2}\right)^{2}+\left(s-2 m^{2}+t\right)^{2}+4 m^{2} t\right],
$$

where $m$ is the electron mass. If we request the bremsstrahlung photon to have a non-infinitesimal energy, there is only one smallness parameter in our problem, namely $\mu=m / E$; we shall now discuss what is the order of $\mu$ of the various invariants in the different collinear situations. To this end, note that with a relative error of $\mathcal{O}\left(\mu^{2}\right)$ we can write

$$
|t|=\frac{m^{2} \Delta_{2}^{2}}{s E E^{\prime}}+E E^{\prime}\left(1-\cos <\left(\vec{q}_{1}, \vec{q}_{2}\right)\right)
$$

where $E^{\prime}=E\left(1-2 \Delta_{2} / s\right)$ is the energy of $q_{2}$. We can therefore distinguish the following collinear situations, in which the cross section displays strong peaks:

- $\mathbf{C}_{1}: \Delta_{1}=\mathcal{O}\left(\mu^{2}\right), \Delta_{2}, t=\mathcal{O}\left(\mu^{0}\right)$;

- $\mathbf{C}_{2}: \Delta_{2}=\mathcal{O}\left(\mu^{2}\right), \Delta_{1}, t=\mathcal{O}\left(\mu^{0}\right)$;

- $\mathbf{C}_{3}: t=\mathcal{O}\left(\mu^{2}\right), \Delta_{1,2}=\mathcal{O}\left(\mu^{0}\right)$;

- $\mathbf{C}_{4}: \Delta_{1,2}=\mathcal{O}\left(\mu^{2}\right), t=\mathcal{O}\left(\mu^{6}\right)$.

Unless $k^{0}$ is very close to $E$, both $s$ and $s_{1}$ are $\mathcal{O}\left(\mu^{0}\right)$. Allowed approximate matrix elements are those in which only the leading orders in $\mu^{-1}$ are kept.

We can now relate Eq.(3) to the old CALKUL result of [4] by noting that in $\mathbf{C}_{1}$ and $\mathbf{C}_{2}$ we may neglect $m^{2}$ with respect to $s, s_{1}$, and $t$, but not to $\Delta_{1,2}$, so we find

$$
\begin{aligned}
\frac{1}{e^{6}}|\mathcal{M}|^{2} \rightarrow & \frac{s^{2}+s_{1}^{2}+z^{2}+z_{1}^{2}}{\Delta_{1} \Delta_{2}(-t)} \\
& -\frac{m^{2}}{\Delta_{1}^{2}} \frac{2\left(s_{1}^{2}+z_{1}^{2}\right)}{t^{2}}-\frac{m^{2}}{\Delta_{2}^{2}} \frac{2\left(s^{2}+z^{2}\right)}{t^{2}}, \\
z= & 2 \Delta_{2}-s-t=\left(p_{1}-q_{2}\right)^{2}+\mathcal{O}\left(\mu^{2}\right), \\
z_{1}= & -2 \Delta_{1}-s_{1}-t=\left(p_{2}-q_{1}\right)^{2}+\mathcal{O}\left(\mu^{2}\right),
\end{aligned}
$$

which is precisely the CALKUL result for these two diagrams. Obviously, this expression is not appropriate to either $\mathbf{C}_{3}$ or $\mathbf{C}_{4}$. We therefore proceed 
to drop, in Eq.(3), terms that are of the order of $m^{2} / \Delta_{1} \Delta_{2} t$, which are always negligible, and we find

$$
\begin{aligned}
\frac{1}{e^{6}}|\mathcal{M}|^{2} & =A_{0}+A_{1}+A_{2}+A_{3}+A_{4}+A_{5}+A_{6}+A_{7}+A_{8}, \\
A_{0} & =\left(s^{2}+s_{1}^{2}+z^{2}+z_{1}^{2}\right) /\left(-\Delta_{1} \Delta_{2} t\right), \\
A_{1} & =-4 m^{2} Z^{2} /\left(\Delta_{1}^{2} \Delta_{2}^{2} t^{2}\right), \\
A_{2} & =-8 m^{2}\left(\Delta_{1}^{2}+\Delta_{2}^{2}\right) /\left(\Delta_{1} \Delta_{2} t^{2}\right), \\
A_{3} & =16 m^{4} Z\left(\Delta_{1}-\Delta_{2}\right) /\left(\Delta_{1}^{2} \Delta_{2}^{2} t^{2}\right) \\
A_{4} & =-16 m^{6}\left(\Delta_{1}-\Delta_{2}\right)^{2} /\left(\Delta_{1}^{2} \Delta_{2}^{2} t^{2}\right) \\
A_{5} & =-4 m^{2} s_{1} /\left(\Delta_{1}^{2} t\right) \\
A_{6} & =-4 m^{2} s /\left(\Delta_{2}^{2} t\right) \\
A_{7} & =-2 m^{2} / \Delta_{1}^{2} \\
A_{8} & =-2 m^{2} / \Delta_{2}^{2} \\
Z & =s \Delta_{1}-s_{1} \Delta_{2}-2 \Delta_{1} \Delta_{2} .
\end{aligned}
$$

Let us tabulate the formal power of $\mu$ of each term in the four distinct collinear situations, writing $\zeta$ for the formal power of $Z$ in $\mathbf{C}_{4}$ :

\begin{tabular}{|c||c|c|c||c|c|c|c|c|c|c|c|c|}
\hline \hline & $\Delta_{1}$ & $\Delta_{2}$ & $t$ & $A_{0}$ & $A_{1}$ & $A_{2}$ & $A_{3}$ & $A_{4}$ & $A_{5}$ & $A_{6}$ & $A_{7}$ & $A_{8}$ \\
\hline $\mathbf{C}_{1}$ & 2 & 0 & 0 & -2 & -2 & 0 & 0 & +2 & -2 & +2 & -2 & +2 \\
$\mathbf{C}_{2}$ & 0 & 2 & 0 & -2 & -2 & 0 & 0 & +2 & +2 & -2 & +2 & -2 \\
$\mathbf{C}_{3}$ & 0 & 0 & 2 & -2 & -2 & -2 & 0 & +2 & 0 & 0 & +2 & +2 \\
$\mathbf{C}_{4}$ & 2 & 2 & 6 & -10 & $2 \zeta-18$ & -10 & $\zeta-14$ & -10 & -8 & -8 & -2 & -2 \\
\hline \hline
\end{tabular}

We note the following points. In order to avoid negative $|\mathcal{M}|^{2}$, we need to have $\zeta \geq 4$ in $\mathbf{C}_{4}$ : we shall see that this is indeed the case. Furthermore, the terms $A_{5,6,7,8}$ are always negligible when $t$ is small. This is a reasonable approximation once we have assumed that the interference between radiation from the $e^{+}$and the $e^{-}$can be discarded. Lastly, we have to ensure that the form (6) can be evaluated in a numerically stable manner. To this end we must control the cancellations inside $Z$, and in addition prove that the matrix element $|\mathcal{M}|^{2}$ is indeed $\mathcal{O}\left(\mu^{-10}\right)$ in $\mathbf{C}_{4}$. These two last items will be solved simultaneously at the end of our discussion. Summarizing the above considerations, we propose the following form for the matrix element, valid 
for small $t$ :

$$
\begin{aligned}
\frac{1}{e^{6}}|\mathcal{M}|^{2}= & \frac{1}{-\Delta_{1} \Delta_{2} t}\left(s^{2}+s_{1}^{2}+\left(s+t-2 \Delta_{2}\right)^{2}+\left(s_{1}+t+2 \Delta_{1}\right)^{2}\right) \\
& -\frac{4 m^{2}}{\Delta_{1}^{2} \Delta_{2}^{2} t^{2}}\left(s \Delta_{1}-s_{1} \Delta_{2}-2 \Delta_{1} \Delta_{2}\right)^{2}-\frac{8 m^{2}}{\Delta_{1} \Delta_{2} t^{2}}\left(\Delta_{1}^{2}+\Delta_{2}^{2}\right) \\
& +\frac{16 m^{4}}{\Delta_{1}^{2} \Delta_{2}^{2} t^{2}}\left(\Delta_{1}-\Delta_{2}\right)\left(s \Delta_{1}-s_{1} \Delta_{2}-2 \Delta_{1} \Delta_{2}\right) \\
& -\frac{16 m^{6}}{\Delta_{1}^{2} \Delta_{2}^{2} t^{2}}\left(\Delta_{1}-\Delta_{2}\right)^{2} .
\end{aligned}
$$

Before proceeding, let us compare the expression of Eq.(7) with existing ones. An earlier form of the matrix element, due to Baier et al. [5] and Altarelli and coworkers [6], reads

$$
\frac{1}{e^{6}}|\mathcal{M}|^{2}=\frac{2\left(s^{2}+s_{1}^{2}\right)}{-\Delta_{1} \Delta_{2} t}-\frac{4 m^{2}}{\Delta_{1}^{2} \Delta_{2}^{2} t^{2}}\left(s \Delta_{1}-s_{1} \Delta_{2}\right)^{2}-\frac{8 m^{2}}{\Delta_{1} \Delta_{2} t^{2}}\left(\Delta_{1}^{2}+\Delta_{2}^{2}\right) \text {. }
$$

We see that Eq.(8) differs from Eq.(7) in that it has a different form for $Z$, and no terms with $m^{4}$ or $m^{6}$ - which, however, cannot be neglected, in contrast to what is commonly the case with ultrarelativistic matrix elements.

An alternative approximation to the matrix element was recently proposed in [1]. It includes terms that have an explicit $m^{2}$, in the following combination, for which we use the notation of the present paper:

$$
\begin{aligned}
G \equiv- & -\frac{4 m^{2}}{\Delta_{1}^{2}}\left(\frac{s_{1}}{t}+\frac{t}{s_{1}}+1\right)^{2}-\frac{4 m^{2}}{\Delta_{2}^{2}}\left(\frac{s}{t}+\frac{t}{s}+1\right)^{2} \\
-\frac{4 m^{2}}{\Delta_{1} \Delta_{2}} & \left(\frac{2 s\left(p_{2}-q_{1}\right)^{2}+3\left(p_{1}-p_{2}\right)^{4}}{t^{2}}\right. \\
& \left.+\frac{s+\left(p_{2}-q_{1}\right)^{2}-2\left(p_{1}-p_{2}\right)^{2}}{t}+1\right) .
\end{aligned}
$$

Let us first note that this expression is not invariant under time-reversal, which in our problem is the operation $p_{1}^{\mu} \leftrightarrow p_{2}^{\mu}, q_{1}^{\mu} \leftrightarrow q_{2}^{\mu}$, and $k^{\mu} \leftrightarrow-k^{\mu}$. What may be even more serious is the peaking behaviour. Expressing $G$ in terms of the five independent invariants $s, s_{1}, t$ and $\Delta_{1,2}$, and retaining only those terms that contribute in $\mathbf{C}_{3}$ or $\mathbf{C}_{4}$, we have

$$
G \sim-\frac{4 m^{2}\left(s \Delta_{1}-s_{1} \Delta_{2}\right)^{2}}{\Delta_{1}^{2} \Delta_{2}^{2} t^{2}}-\frac{48 m^{2}\left(\Delta_{1}-\Delta_{2}\right)^{2}}{\Delta_{1} \Delta_{2} t}+\frac{16 m^{2} s\left(\Delta_{1}-2 m^{2}\right)}{\Delta_{1} \Delta_{2} t^{2}} .
$$


The first two of these terms are $\mathcal{O}\left(\mu^{-10}\right)$ in $\mathbf{C}_{4}$, but the last is $\mathcal{O}\left(\mu^{-12}\right)$, since in general the combination $\Delta_{1}-2 m^{2}$ is $\mathcal{O}\left(\mu^{2}\right)$ rather than $\mathcal{O}\left(\mu^{4}\right)$. We therefore have to conclude that, at least in $\mathbf{C}_{4}$, the result of [1] is not appropriate. Note, however, that in the soft-photon limit disucssed in [1], $\Delta_{1} \rightarrow 0$ so that the correct $\mathcal{O}\left(\mu^{10}\right)$ behaviour is recovered in that case.

Let us now turn to the numerical stability of the expression (7). We may assume that we can evaluate $\Delta_{1,2}$ and $t$ in a stable manner for given momenta. It remains to control the cancellation from $\mathcal{O}\left(\mu^{2}\right)$ down to $\mathcal{O}\left(\mu^{4}\right)$ inside $Z$, that occurs in $\mathbf{C}_{4}$. To this end we introduce $y=q^{0} / E, u=1-y$, and a vector $r^{\mu}$, by $q_{2}^{\mu}=u q_{1}^{\mu}+r^{\mu}$. Denoting by the subscript $L$ components along $\vec{q}_{1}$, and by $T$ the orthogonal components, we find

$$
\begin{aligned}
r^{\mu} & =\left(r^{0}, \vec{r}_{T}, r_{L}\right) \\
r^{0} & =0 \\
\vec{r}_{T} & =-\vec{q}_{T} \\
r_{L} & =\left(-2 m^{2} y+t\right) /(2 p) \\
r \cdot r & =m^{2} y^{2}+u t
\end{aligned}
$$

with $p=\left|\vec{p}_{1}\right|$. In $\mathbf{C}_{4}$, then, $\vec{r}_{T}$ is $\mathcal{O}\left(\mu^{3}\right)$, and $r_{L}$ is $\mathcal{O}\left(\mu^{4}\right)$, since $y=\mathcal{O}\left(\mu^{2}\right)$. Some straightforward algebra now leads to the surprisingly simple form

$$
Z=s(k \cdot r)
$$

The best frame to evaluate this expression is the rest frame of $R^{\mu} \equiv p_{2}^{\mu}+k^{\mu}$. Denoting the various components in this frame by carets, we have, with $w=\sqrt{R \cdot R}=\mathcal{O}\left(\mu^{2}\right)$ :

$$
\begin{aligned}
& \hat{r}^{0}=-\left(2 m^{2} y+t\right) /(2 w), \\
& \hat{\vec{r}}_{T}=-\vec{q}_{T}\left(1+\frac{\hat{r}^{0}}{R^{0}+w}\right), \\
& \hat{r}_{L}=r_{L}+\left(p-q_{L}\right) \frac{\hat{r}^{0}}{R^{0}+w}, \\
& \hat{k}^{0}=\left(w^{2}-m^{2}\right) /(2 w) .
\end{aligned}
$$

we see that all components of $\hat{r}^{\mu}$ are $\mathcal{O}\left(\mu^{3}\right)$, and $\hat{k}^{\mu}$ is $\mathcal{O}(\mu)$. Since $r \cdot r$ is 
$\mathcal{O}\left(\mu^{6}\right)$, no further cancellations are to be expected

Finally, we prove that, in $\mathbf{C}_{4}$, the matrix element is indeed $\mathcal{O}\left(\mu^{-10}\right)$. In this matrix element, the electron line and the virtual photon propagator enter via the object

$$
\begin{aligned}
H^{\mu \nu}= & \frac{1}{t^{2}} \sum_{\text {spins }} \bar{v}\left(q_{1}\right) \gamma^{\mu} u\left(q_{2}\right)\left(q_{2}\right) \gamma^{\nu} v\left(q_{1}\right) \\
& \frac{4}{t^{2}}\left(q_{1}^{\mu} q_{2}^{\nu}+q_{1}^{\nu} q_{2}^{\mu}+\frac{t}{2} g^{\mu \nu}\right) .
\end{aligned}
$$

The last term cancels one of the factors $t$, and will hence not contribute in $\mathbf{C}_{4}$. We now rewrite $q_{1,2}^{\mu}$ in terms of $q^{\mu}$ and $r^{\mu}$ :

$$
q_{1}^{\mu}=\left(r^{\mu}+q^{\mu}\right) / y \quad, \quad q_{2}^{\mu}=\left(r^{\mu}+u q^{\mu}\right) / y
$$

Owing to current conservation, the terms with $q^{\mu}, q^{\nu}$ evaluate to zero, and the relevant part of the electron current is therefore

$$
H^{\mu \nu}=\frac{8 r^{\mu} r^{\nu}}{y^{2} t^{2}}
$$

This object, when evaluated in the $R^{\mu}$ rest frame, is itself of order $\mathcal{O}\left(\mu^{-10}\right)$ as we have seen above. In addition, again in the $R^{\mu}$ rest frame, the positron current is $\mathcal{O}\left(\mu^{0}\right)$ since there

$$
\begin{aligned}
p_{1,2}^{\mu}, k^{\mu} & =\mathcal{O}(\mu), \\
\Delta_{1,2} & =\mathcal{O}\left(\mu^{2}\right), \\
u\left(p_{1,2}\right) & =\mathcal{O}\left(\mu^{1 / 2}\right) .
\end{aligned}
$$

This finishes the argument: $|\mathcal{M}|^{2}$ is indeed $\mathcal{O}\left(\mu^{-10}\right)$ in $\mathbf{C}_{4}$, and we can evaluate it in a numerically stable manner, without the need for extended precision $(\mathrm{REAL} * 16)$ as in [1] to control the cancellations. An application of the form (7), to the study of beam-beam-strahlung as a limitation to the LEP beam lifetimes, will be presented elsewhere [7].

\footnotetext{
${ }^{1}$ If $r^{\mu} r_{\mu}$ was smaller, yet another cancellation might be lurking in the product $k \cdot r$.
} 


\section{References}

[1] M. Greco, G. Montanga, O. Nicrosini and F. Piccinini, preprint FNT/T-93/20 and LNF-93/035(P).

[2] H. Burkhardt, unpublished.

[3] J.A.M. Vermaseren, Symbolic Manipulation with FORM, published by Computer Algebra Nederland, Amsterdam, the Netherlands, ISBN 90-74116-01-9.

[4] F.A. Berends, R. Kleiss, P. de Causmaecker, R. Gastmans, W. Troost, and T.T. Wu, Nucl.Phys.B206 (1982) 61;

R. Gastmans and T.T. Wu, The Ubiquitous Photon: Helicity Method for QED and QCD, Oxford Science Pub., 1990.

[5] V.N. Baier, V.S. Fadin, V.A. Khoze, Sov. Phys. JETP 24 (1967) 760; 26 (1968) 1238.

[6] G. Altarelli and F. Bucella, Nuovo Cim. 34 (1964) 1337;

G. Altarelli and Stella, Nuovo Cim. 34 (1964) 1337.

[7] H. Burkhardt and R. Kleiss, in preparation. 